\title{
A Human Expert Based Approach to Heartrate Monitoring System with Automatic Message Sending Via Smartphones
}

\author{
Dr. J. Arun ${ }^{1}$, Saranya. $\mathrm{N}^{2}$ \\ ${ }^{1}$ Head of the Department,Computer Science and Engineering,Dhanalakshmi Srinivasan Engineering College, \\ Perambalur, Tamil Nadu, India \\ ${ }^{2}$ ME-Computer Science and Engineering, Dhanalakshmi Srinivasan Engineering College, Perambalur, Tamil \\ Nadu, India
}

\begin{abstract}
Article Info

Volume 7, Issue 2

Page Number : 401-405

Publication Issue :

March-April-2021

\section{Article History}

Accepted : 15 April 2021

Published : 22 April 2021

The objective of the paper was to propose a human - expert based approach on a heartbeat calculating and message sending system. Heart related disease is rising among today's population. The need for an accurate yet affordable heart rate is essential to ensure one's health quality. However, in today's market, most heart monitors are expensive and are not user friendly. Our goal is to calculate the heart beat using smart phones that is user friendly and less expensive. In Today's situation $50 \%$ percent of people using Smart phones, so in order to make our goal possible we are using the smart phones to calculate the humans heart beat rate accurate. In this project, a real-time heart rate measurement technique called Photo-Plethysmography (PPG) is implemented using simple infrared transmitter and receiver circuit. Our main aim to decrease the death rate of humans due to heart related diseases, so we can assign the smart phones to sending an emergency alert message to the particular persons relatives and to an ambulance or to the nearby doctor resources along with the location of the user when the Blood pressure is increasing/decreasing then normal rate. By sending an alert message the lives of an human will be recovered from critical situation.

Keywords : Heart Beat Sensor, Capacitive Sensor, Monitoring, Cardiac Diseases, Mortality Rate.
\end{abstract}

\section{INTRODUCTION}

Heart beat rate is defined as the number of heartbeats per unit time, that is expressed as beats per minute (bpm). The human heart pounds to pump oxygen rich blood to the muscles and carry waste products of cell away from the tissues. Heartbeat rate can vary significantly between individuals based on age, fitness and genetics. It also varies according to the demand of the muscles to absorb oxygen and excrete carbon dioxide changes such as during exercise or sleep. Heart rate is varying according to age, person physical and activity condition. Human heart rate for adult that healthy is around 60 to 100 beats per minute (bpm). It is developed to give patients a timely and proper heath care. Heart rate measurement could show the condition of the heart. While for an athlete, their heart rate is slower than an active adult. For baby on the other hand, the heart rate is higher which is around 120 to $160 \mathrm{bpm}$ and for their children's heart rate is around 75 to $110 \mathrm{bpm}$. Abnormal heart rate such as lower heart rate than the

Copyright: @ the author(s), publisher and licensee Technoscience Academy. This is an open-access article distributed under the terms of the Creative Commons Attribution Non-Commercial License, which permits unrestricted non-commercial use, distribution, and reproduction in any medium, provided the original work is properly cited 
normal rate is called bradycardia whereas for a higher heart rate which is higher than the normal is called tachycardia. The traditional heart rate is measurable by putting finger above pulse artery and count pulse rate within 30 second and heart rate (bpm) can be found with multiply with. This way is easy but inaccurate especially when artery pulse state in high rate. The most accurate method to measure heart rate is by using electrocardiogram machine (ECG) but this equipment expensive and not affordable by individuals. The goal of our project is to design a less expensive heart rate monitor will provide an accurate reading of one's heart rate. It will measure the heart rate from any part of a human body using Heart Beat sensor to detect changes in blood flow. The heart rate will be displayed on an LCD display of our smart phones for easy monitoring. The significance of the heart monitor is that it provides an inexpensive and accurate means of measuring one's heart rate at his/her convenience.

\section{Statement of the problem}

The human health is one of the most important concerns in the world today. Everything becomes meaningless when human becomes sick and dies due to improper medical care. For health reasons, governments, people and several organizations spend a lot of money to ensure a better health condition for themselves and the entire population. Scientists and Engineers are always at work to device a means of supporting and sustaining a sound health condition for all through the invention of numerous technologies both Electrical/Mechanical gadgets that are in use for health care delivery nowadays. The heart is a very cardinal organ in the human body (once it stops beating, nothing else matters). Thus, if early actions are taken on time then the heart condition can be managed effectively and many patients can be cured from their problems and saved. The problem of patients slumping and dying is associated with cardiovascular arrests and can be checkmated this sensitive and highly effective application (the patient heartbeat monitor). This application has an outstanding advantage that it is easy to handle and access. The development of a userfriendly application to monitor heart beats using infrared sensor will be the foundation for an inexpensive heart rate monitor. This prototype would benefit the users to monitor their heart rate at home or any place.

\section{Objective}

The main objective of the project is to design and develop a low-cost portable heart rate monitor as an android application, in that the designed system updates the user with heart rate and sends a Short Message Service in case of an emergency. The system calculates the rate of Heartbeat and sends alert messages to family members, everything just at a click of a button. Android studio is the tool used to develop the application. Photo plethysmography is based on the principle that blood absorbs more light than the surrounding tissue. In addition, variations in blood volume (systole and diastole) affect the transmission or reflectance of light. These two principles can be used to detect blood flow. By using this system and checking our health on daily basis it is possible to reduce the chance of death due to heart related diseases.

The prototype is a low-cost portable device that can calculate and display the heart rate by count the pulse generate by the sensor. The heartbeat rate is measured from the fingertip placed in smartphones back camera lens. The generated heartrate is the output of the sensor where the flow of blood is absorbed and the heartrate is calculated by the reflected rays. The heart rate is displayed on a smartphone display and the alert message is send to the emergency contacts which is stored in the 
database. Today in the current global scenario, the prime question in every human's mind, considering the ever-rising increase of issues on heart related diseases. An android based application needs to be developed, in order to intimate the emergency situations of the patients. Aim of the project is to decrease the mortality rate of the people affected by heart related diseases, especially the people those are living alone. The proposed model is much more precise, straightforward and cheaper than other heartbeat rate measuring systems. This work has tried to make an easy and stout system for the monitoring of heartbeat.

\section{Abbreviations and Acronyms}

\section{ECG-Electro Cardio Gram}

BPM-Beats per Minute

LCD-Liquid Crystal Display

PPG- Photo-Plethysmography

\section{METHODOLOGY}

The proposed system is to plan a compact device to diagnosis the heart rate efficiently. The Principle of Heartbeat Sensor is used to measure the Heart rate of the human body. The heartbeat sensor is based on the principle of photoplethysmography. It measures the change in blood flow through any organ of the body that causes a change in the light intensity through that organ.

Using a programming language to connect the Heartbeat sensor and capacitive sensor to calculate the Heart rate, at the time the blood vessels are abnormal to the normal flow of blood. The basic heartbeat sensor consists of a light emitting diode and a detector like a light detecting resistor or a photodiode. The heart beat pulses cause a variation in the flow of blood to different regions of the body. If a tissue is illuminated with the light source, i.e., light emitted by the led, it either reflects (a finger tissue) or transmits the light. Some of the light is absorbed by the blood and the transmitted or the reflected light is received by the light detector. The amount of light absorbed depends on the blood volume in that tissue. The detector output is in form of electrical signal and is proportional to the heart beat rate.

This study develops a heart beat monitoring framework to detect underlying heart conditions in realtime which helps avoiding potential heart diseases and rehabilitation of the patients recovering from cardiac diseases. The proposed real-time monitoring system is compatible and portable to use various sensors to extract medical information which helps finding out heart rate. These cardiac parameters help early detection of diseases such as arrhythmia, hypotension, hypertension, and hyperthermia through the detecting system based on upper and lower threshold values.

\section{Working}

Principle of Heartbeat Sensor. Heartbeat sensor is used to measure the Heart rate of our human body. The heartbeat sensor is based on the principle of photo phlethysmography. It measures the change in blood flow through any organ of the body which causes a change in the light intensity through that organ (a vascular region).Using a programming to connect the Heartbeat sensor and capacitive sensor to calculate the Heart rate when the blood vessels are abnormal to the normal flow of blood. The basic heartbeat sensor consists of a light emitting diode and a detector like a light detecting resistor or a photodiode. The heart beat pulses causes a variation in the flow of blood to different regions of the body. When a tissue is illuminated with the light source, i.e. light emitted by the led, it either reflects (a finger tissue) or transmits the light (earlobe). Some of the light is absorbed by the blood and the transmitted 
or the reflected light is received by the light detector. The amount of light absorbed depends on the blood volume in that tissue. The detector output is in form of electrical signal and is proportional to the heart beat rate.

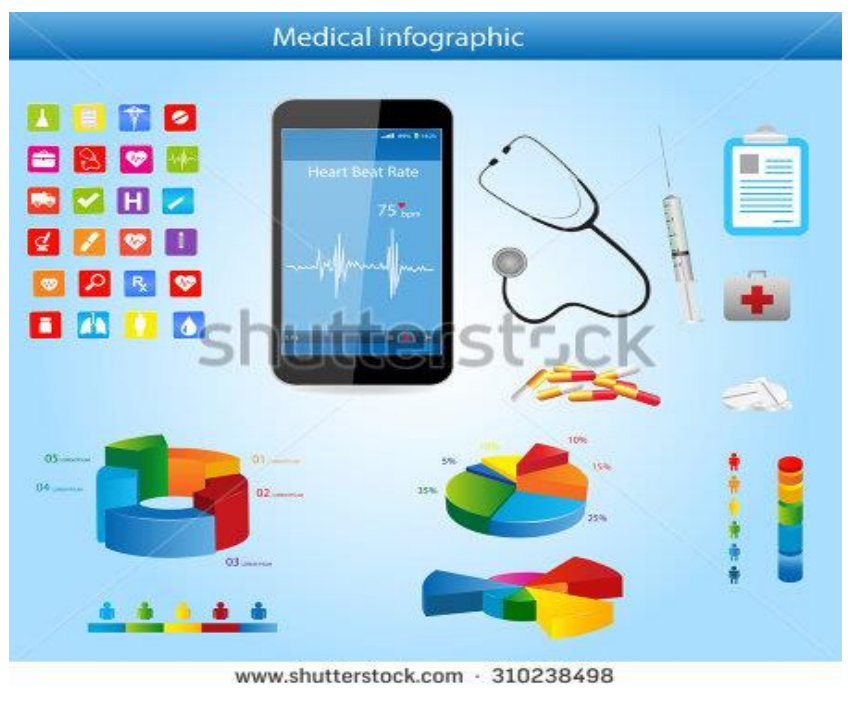

In capacitive sensor screens are made from multiple layers of glass. The inner layer conducts electricity and so does the outer layer, so effectively the screen behaves like two electrical conductors separated by an insulator-in other words, a capacitor. Capacitive screens can be touched in more than one place at once. Unlike most other types of touch screen, they work if you touch them with anyplace of the LCD screen. They don't work if we touch them with plastic hands (because the plastic is an insulator and stops your hand from affecting the electric field).

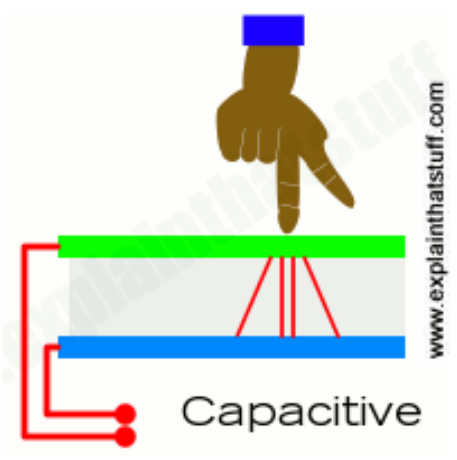

These two sensors have been controlled and monitored to calculate the heart beat rate. When there is an abnormal increase/decrease in the human heart beat rate, automatic message have been send to the nearby ambulance, close relative and to the hospital source for the first aid purpose. While people driving anywhere or they are alone in home, if suddenly heartbeat rate increase/decrease it sends message automatic to these three important persons. And the open-source map has been attached to the application which is used to find the location of the corresponding person who are in a critical situation. So, we will easy to help the person while using these sensors in any critical situations. So, it is very helpful to decrease the death rate among the people. Suppose if there are no medical facilities in the nearby source, the first aid has been provided by the doctor consultant. By providing the first aid, the patient's health will be saved for some more times. The main aim of our application is to decrease the death rate of human by monitoring the heart beat rate of a human continuously and by sending a alert message when there is a abnormal increase/decrease in the heart beat rate to the relatives and to the ambulance and to the nearby hospital source suppose when there they are far away from the hospital source the first aid has been provided by the doctor consultant.

\section{Figures}

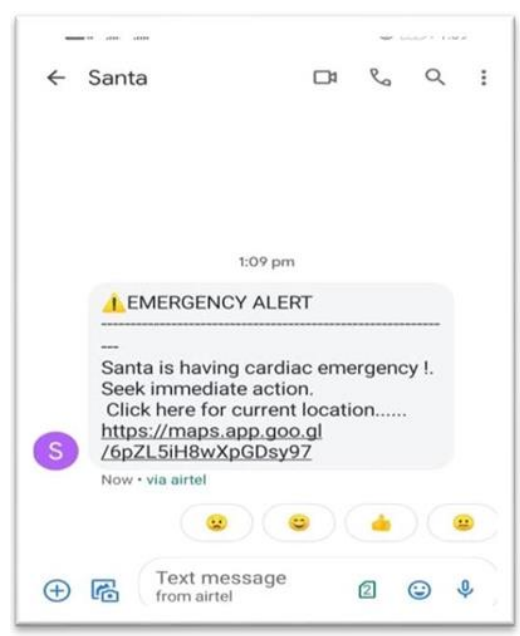




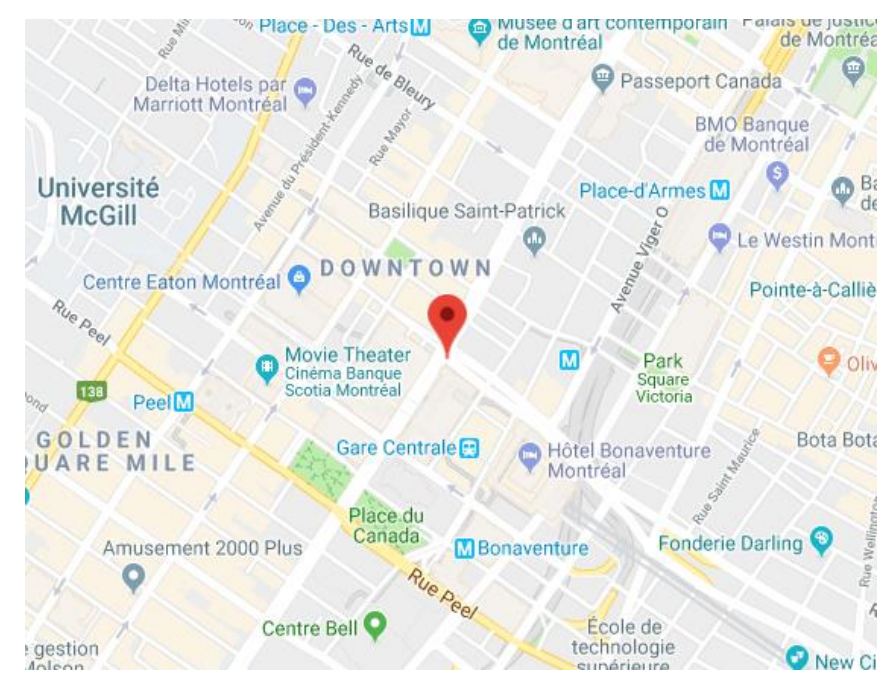

Alert message along with the location is send in case of emergency

\section{Features}

$>$ Continuous monitoring of the heart beat rate.

$>$ Alert message when in abnormal heart beat rate.

$>$ First aid have been provided in a critical situation.

$>$ Accuracy and Convenience.

$>$ Decreasing the death rate due to cardiac diseases.

$>$ Easy to monitor

\section{CONCLUSION AND FUTURE ENHANCEMENT}

The thousands of hardware devices are available in the market lack in some features such as message sending and location sending. In the present era, the developed application is very helpful for all people those having heart related diseases. By simple on touch the application, it will automatically calculate the heart rate, alert the guardians if the person is in emergency situation. Simply touching the application, the application will send the location information as a message to the all-saved guardian contacts. Through this application, can alert the people at remote areas, if the person feels palpitations and bradycardia.

In today's increasing population, heart related diseases also increase rapidly. Heart beat monitoring application has been chosen by considering so many factors that involve saving lives. In future, artificial intelligence and machine learning can be implemented in the application in order to diagnose the user's problem accurately from the user's description about the problem. patient's data such as prescriptions, operation details, scans, etc., can also be made to store in a common database so that it can be accessed any place any time.

\section{REFERENCES}

[1]. Ajit Kumar Patro, Bandana Mallick , (2016) "Heart rate monitoring system using fingertip through arduino and processingsoftware".

[2]. Ato.Biniyam, Ato.GutaTesema and Ato.AkremMohammed ,(2017)“ Design and implementation of heart beat monitoring using PIC microcontroller “.

[3]. BhagyaLakshmi.J.M, Hariharan.R, UdayaSri.CNandhiniDevi.P,

Sowmiya.N,(2015)“Heartbeatdetectorusinginfraredp ulsesensor".

[4]. Ch.Sandeep Kumar Subudhi, (2014) "Intelligent wireless patient monitoring and tracking system (using sensor network and wireless communication)".

[5]. Hashem et al., (2010)"Design and development of a heart rate measuring device usingfingertip".

\section{Cite this article as :}

Dr. J. Arun, Saranya. N, "A Human Expert Based Approach to Heartrate Monitoring System with Automatic Message Sending Via Smartphones", International Journal of Scientific Research in Computer Science, Engineering and Information Technology (IJSRCSEIT), ISSN : 2456-3307, Volume 7 Issue 2, pp. 401-405, March-April 2021. Available at doi : https://doi.org/10.32628/CSEIT217271 Journal URL : https://ijsrcseit.com/CSEIT217271 